\title{
Fractal-Based Approach for Segmentation of Address Block in Postal Envelopes
}

\author{
Luiz Felipe Eiterer ${ }^{1}$, Jacques Facon ${ }^{1}$, and David Menoti ${ }^{1,2}$ \\ 1 PUCPR-Pontifícia Universidade Católica do Paraná \\ Rua Imaculada Conceição 1155, Prado Velho \\ 80215-901 Curitiba-PR, Brazil \\ \{eiterer,facon,menoti\}@ppgia.pucpr.br \\ ${ }^{2}$ UFMG Universidade Federal de Minas Gerais \\ DCC Departamento de Ciência da Computação
}

Av. Antônio Carlos, 6627 31.270-010, Belo Horizonte-MG, Brazil

menoti@dcc.ufmg.br

\begin{abstract}
In this paper, an address block segmentation approach based on fractal dimension $F D$ is proposed. After computing the fractal dimension of each image pixel by the $2 \mathrm{D}$ variation procedure, a clustering technique based on K-means is used to label pixels into semantic objects. The evaluation of the efficiency is carried out from a total of 200 postal envelope images with no fixed position for the address block, postmark and stamp. A ground-truth strategy is used to achieve an objective comparison. Experiments showed significant and promising results. By using the $2 \mathrm{D}$ variation procedure for three ranges of neighbor window sizes $(r=\{3,5\}, r=\{3,5,7\}$, and $r=\{3,5,7,9\})$, the proposed approach reached a success rate over than $90 \%$ on average.
\end{abstract}

\section{Introduction}

It is well known that the proper addressing allows mail pieces to be processed quickly and more efficiently. We also know that this task is not a mystery. When the mail piece meets size requirements, address block and zip code are filled in correctly and the proper amount of postage is obeyed, it moves easily through the mechanized sorting process saving labor and time. Therefore, despite of all rules mentioned above, why is so difficult to increase mail sorting automation? Some reasons like wide variety of postal envelope attributes (layouts, colors and texture), the handwritten address block which appears mixed up with postmarks or stamps are factors that increase the complexity of an efficient mail sorting system.

Several authors have dealt with the problem of locating address blocks in envelope images. For instance, Yu et al in [1] present a method for locating address blocks from magazine envelopes based on the following assumptions: The address is written on a light-colored label, generally skewed. Moreover, the label may be stuck on the magazine, under the plastic envelope or displayed in a plastic window provided in the envelope. The address block follows the left alignment rule. The contrast between the ink and the spaces between characters varies according to the equipment used (laser or matrix printer, or even a typewriter). And the magazine and envelope ensemble contains other text messages in addition to the address block. The authors segment magazine envelopes using an Otsu's modified method which reduces the influence of the grayscale 
distributions. Then a connected component analysis based on $B A G$ strategy is used to identify individual blocks of text. Some heuristics are used to eliminate false address block candidates. Finally, a recognizer is used to locate the address block. Experiments on 53 IBM magazine envelopes and 52 other ones showed that the method was successful in locating the correct address block in $71.70 \%$ and $92.86 \%$, respectively.

Recently, Yonekura et al [2] present a method for postal envelope segmentation combining the 2-D histogram and morphological clustering. A new filter based on the morphological grayscale dual reconstruction is proposed to the 2-D histogram calculation and the proposed clustering is based on the watershed transform criterion. Experiments on a database composed of 300 complex postal envelope images, with creased background and no fixed position for the handwritten address blocks, postmarks and stamps showed that the method was successful in locating the correct address block in $75 \%$.

The goal of this paper is to propose and to evaluate a new postal envelope segmentation method based on Fractal Dimension $F D$ clustering. The aim is to locate and segment handwritten address blocks, postmarks and stamps with no a priori knowledge of the envelope images (Figure 1). The Fractal Dimension clustering is carried out by means of the K-means. A ground-truth segmentation is employed to evaluate the accuracy of this approach.

This paper is organized as follows: Section 2 shortly reviews fractal-based techniques to solve the segmentation challenge. Section 3 describes the grayscale image fractal dimension method used in our approach. The proposed method based on the $\mathrm{K}$-means is detailed in Section 4. Section 5 presents some experimental results and discussions. In addition, the proposed segmentation method is evaluated by means of ground-truth images in Sections 5.1 and 5.2. Finally, some conclusions are drawn in Section 6.

\section{Fractal-Based Techniques for Segmentation Challenge}

Fractals are very useful in modeling roughness and self-similarity properties of surfaces in Image Processing. The fractal dimension gives a measure of the roughness of

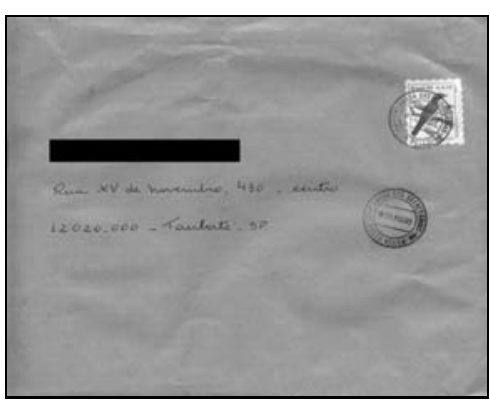

(a)

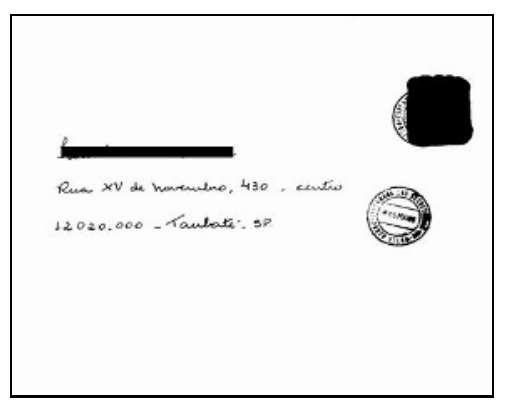

(b)

Fig. 1. Example of envelope image segmentation: (a) Envelope image, (b) Ground-truth handwritten address block, postmark and stamps 
a surface. Intuitively, the larger the fractal dimension, the rougher the texture is. Some authors have developed recent practical applications in segmentation challenges. For instance, Diayana et al [3] have compared three microcalcification detection methods in digital mammogram. They showed that the fractal analysis based on morphological dilation and erosion in $8 \times 8$ image blocks is a promising approach.

Andrew Ringler [4] has used a local fractal dimension $F D$ approach to segment oceanic images into water and no-water classes. By using the $2 \mathrm{D}$ variation procedure for each image pixel, the author has successfully segmented ocean waves to detect dolphin and swimmers and to perform human search and rescue.

Samarbandu et al [5] have proposed an initial study of bone X-Rays images through a morphological fractal analysis. This $F D$ approach, where only the dilation is used, has demonstrated the feasibility of detecting bone structures independent of scale.

\section{Fractal Dimension for Grayscale Images}

Since the nature of textured surfaces is not deterministic, the computation of grayscale image fractal dimension $F D$ is not an easy task. Some methods were proposed for estimating the $F D$ for grayscale images. To better understand how the fractal dimension $F D$ can be computationally estimated, let first explain the most popular method, the $D B C$ differential box-counting method [6]. Consider an $M \times M$ pixel image as a surface in $(x, y, z)$ space where $P(x, y)$ represents the pixel position and $z$ is the pixel intensity. One can partition the $(x, y)$ space into a grid of $s \times s$ size pixels. An estimate of the relative scale is $r=s / M$. At each grid position, one stacks cubes of size $s$, numbering each box sequentially from 1 up to the box containing the highest intensity in the image over the $s \times s$ area. Denoting the minimum and maximum gray levels for the image at position $(i, j)$ by $k$ and $l$ respectively, one can define $n_{r}(i, j)=l-k+$ 1. A parameter $N(r)$ is then estimated by summing over the entire grid as $N(r)=$ $\sum_{(i, j)} n_{r}(i, j)$. The above procedure is repeated for a number of values of $r(s)$. The slope estimation of the graph $\log (N(r))$ versus $\log (1 / r)$ by the least-squares method provides the fractal dimension $F D$.

Among the Fractal-based approaches available in literature, one could observe that the $2 \mathrm{D}$ variation procedure is not very complex and has shown to be promising in complex images (see [4]). It is the reason why it has been chosen. The $2 \mathrm{D}$ variation procedure, proposed by [7], similar to the differential box-counting method, analyses the pixel environment at different distances $r$. The squares with different sizes $r$ are running pixel by pixel across the image from left to right and from top to bottom. The algorithm determines the minimum min and maximum max gray values within the square of size $r$. Since the intensity value $v$ of a pixel $p$ is related to the height of the objects in the image, one can meaningfully define at $p$ a volume $r \times r \times v$. By defining the difference volume of $p$ as $r \times r \times(\max -\min )$, one can denote $V(r)$ as the sum of differences between the maximum and minimum for scale $r$ for the entire image, resulting in a power law: $V(r)=$ const $r^{s}$. In the Richardson-Mandelbrot plot $(\log (V(r))$ versus $\log (r))$, the dependence of this volume $V(r)$ should be linear with the square size $r$. By using the least-squares method to this linear regression, one can compute the slope $s$ of this line and then deduce $F D$ : 


$$
F D=3-s / 2
$$

We applied the 2D variation procedure onto postal envelope images for three ranges of neighbor window sizes $(r=\{3,5\}, r=\{3,5,7$,$\} , and r=\{3,5,7,9\})$. It results in an image $I_{F D}$ where the $F D$ of each pixel stays in the range [2.0,3.0] (see Equation 1).

\section{Clustering}

We are aiming at automatic clustering, where the handwritten address block, postmark and stamp classes should be extracted from the image through their fractal dimension. For this purpose, we use the K-means clustering which is a robust unsupervised algorithm often applied to the problem of image texture segmentation. The K-means clustering is an algorithm for partitioning $N$ data points into $k$ disjoint clusters $C_{j}$ containing $N_{j}$ data points so as to minimize the sum-of-squares error. Each cluster in the partition is defined by its member data points and by its centroid. The centroid for each cluster is the point to which the sum of distances from all data points in that cluster is minimized. The K-means clustering uses an iterative algorithm that minimizes the sum of distances $S_{d}=\sum\left[C_{j}(P)-P\right]^{2}$ from each data point $P$ to its cluster centroid $C_{j}$, over all clusters. This algorithm moves data points between clusters until the sum cannot be decreased further. The result is a set of clusters that are as compact and well-separated as possible. It is comprised of a simple re-estimation procedure as follows [8] [9]:

1. First, the data points are assigned at random to the $k$ sets;

2. Second, the centroid is computed for each set.

These two steps are alternated until a stopping criterion is reached (i.e. when there is no further change in the assignment of the data points). Details of the minimization can be controled using several optional input parameters to K-means, including those for the initial values of the cluster centroids, and for the maximum number of iterations.

The input parameters used in our K-means clustering approach are:

- Number of features: only one, the $F D$ itself;

- Number of clusters : 3 clusters which are the handwritten address block, postmark and stamp one, the background one and the noise one;

- Number of iterations: 10;

- Convergence criterion: 0.0001 .

It is well-known that the K-means algorithm suffers from initial starting conditions effects (initial clustering effects). One can find in literature some researches about the initialization optimisation of iterative partitional clustering [10]. In our approach, min, $(\max +\min ) / 2$ and $\max$ are used to initialize the values of cluster centroids, where $\max$ and $\min$ are the $F D$ buffer maximum and minimum values, respectively. The convergence of the K-means algorithm onto the image $I_{F D}$ results in the labeled image $I_{K \text { means }}$ where each pixel is labeled into the 3 clusters. 


\section{Experiments}

A database composed of 200 complex postal envelope images, with no fixed position for the handwritten address blocks, postmarks and stamps was used to evaluate the efficiency of the proposed approach. Each image has about $1500 \times 2200$ pixels and was digitized at $300 \mathrm{dpi}$. We could verify that the address blocks, stamps and postmarks represent only $1.5 \%, 4.0 \%$ and $1.0 \%$ of the envelope area, respectively and that the great majority of pixels of these images belong to the envelope background (approximatively $93.5 \%)$.

\subsection{Evaluation Strategy of the Proposed Method}

A ground-truth strategy was employed to evaluate the accuracy of the proposed approach. The ideal result (ground-truth segmentation) regarding the handwritten address block, postmark and stamp class has been generated for each envelope image (Figure 1(b)). The comparison between the ground-truth segmentation and the results obtained by the proposed approach was carried out pixel by pixel. We have computed each obtained result with the ideal segmentation in terms of identical pixels at the same location. We have also computed the average of all obtained results for each class.

\subsection{Numerical Results and Discussion}

Figures 2 and 3 show interesting address block, stamp, postmark segmentations in case of envelope images with creased background and wrong layout (Figure 2-(a)), with many stamps and postmarks (Figure 2-(b)), and without stamp (Figure 3). In spite of the background complexity, and independently of the semantic classes, the address blocks, stamps and postmarks are very well segmented. As explained before, the evaluation of the approach was carried out by comparing pixel by pixel the ground-truth segmentation and the results obtained.

The average segmentation rates for each class are showed in Table 1. This table shows how the accuracy of the algorithm changes if the box size range $r$ is changed. Best values are obtained for range $r=\{3,5,7,9\}$, where the segmentation successfully recovered address blocks $(97.24 \% \pm 13.64 \%)$ postmarks $(91.89 \% \pm 17.22 \%)$. The most important figures are the ones related to the address block, since for practical purposes the classes of stamp, and postmark would be discarded in the end and it would not harm the result. It is possible to conclude that, independently of the layout and background in the input images, the segmentation recovered the address blocks and postmarks with great success. It can be seen that the segmentation did not recovered the stamps as expected. It is caused by the fact that the stamps contain some complex drawings, gray level differences (dark objects and bright background).

\section{Conclusions}

The use of fractal dimension in postal envelope segmentation was proposed. After computing the fractal dimension for each image pixel, the resulting fractal image is then 


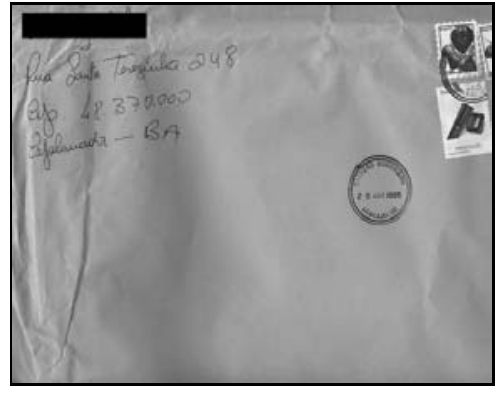

(a)

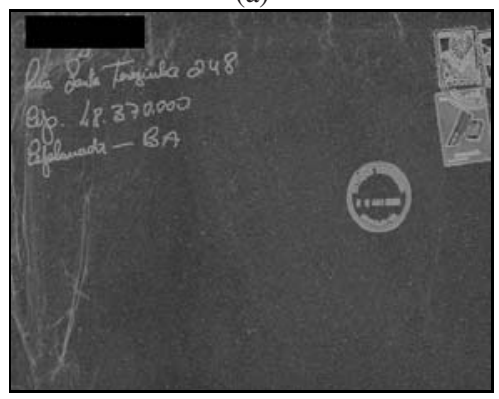

(c)

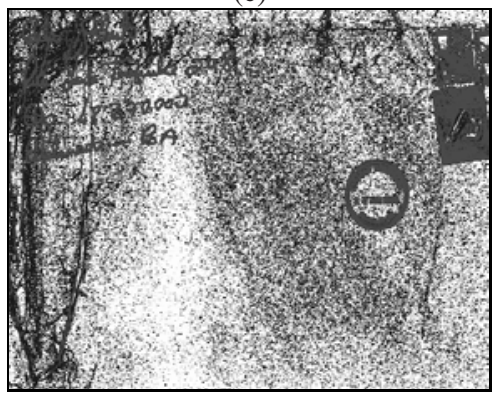

(e)

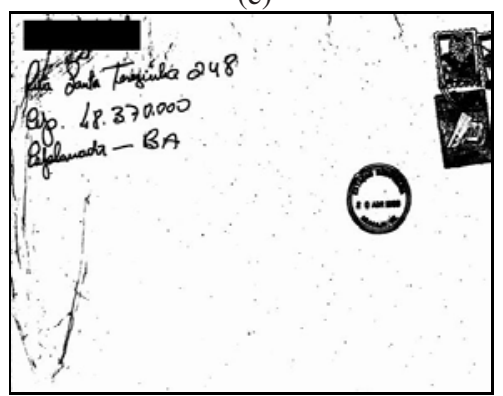

(g)

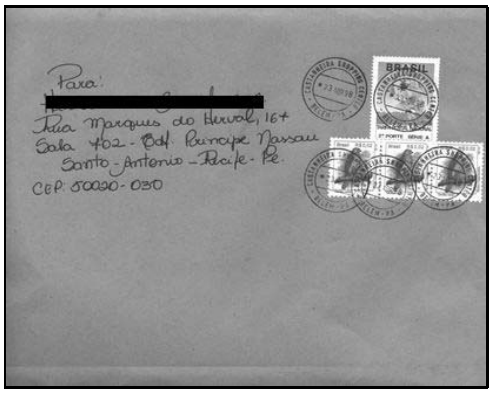

(b)

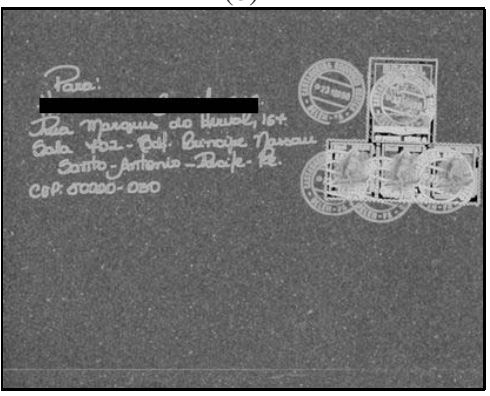

(d)

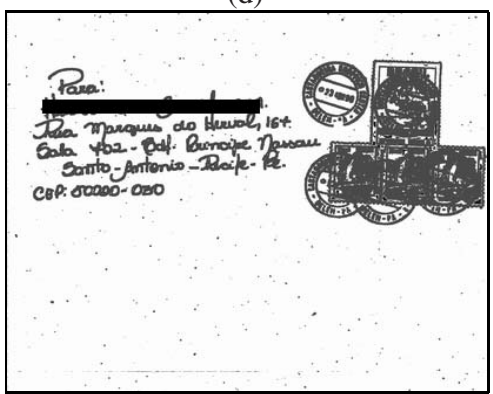

(f)

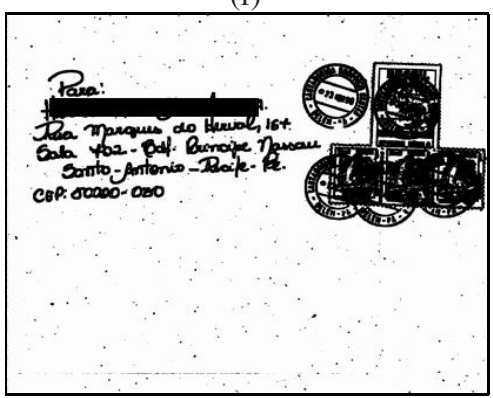

(h)

Fig. 2. Results obtained to different envelope images: (a) with creased background and wrong layout, (b) with many stamps and postmarks: (c)-(d) Fractal Images, (e)-(f) K-means clustering, (g)-(h) Handwritten address blocks, postmarks and stamps 
Table 1. Average results with identification of regions (pixel by pixel accuracy)

\begin{tabular}{l|c|c|c} 
& \multicolumn{3}{|c}{ Accuracy pixel by pixel $(\mu \pm \sigma)$} \\
\hline Box sizes & $r=\{3,5\}$ & $r=\{3,5,7\}$ & $r=\{3,5,7,9\}$ \\
\hline Address Block & $92.61 \% \pm 13.61 \%$ & $96.05 \% \pm 10.89 \%$ & $97.24 \% \pm 13.64 \%$ \\
\hline Stamp & $48.86 \% \pm 13.84 \%$ & $59.76 \% \pm 15.68 \%$ & $66.34 \% \pm 16.90 \%$ \\
\hline Postmark & $82.02 \% \pm 19.66 \%$ & $88.97 \% \pm 17.58 \%$ & $91.89 \% \pm 17.22 \%$
\end{tabular}

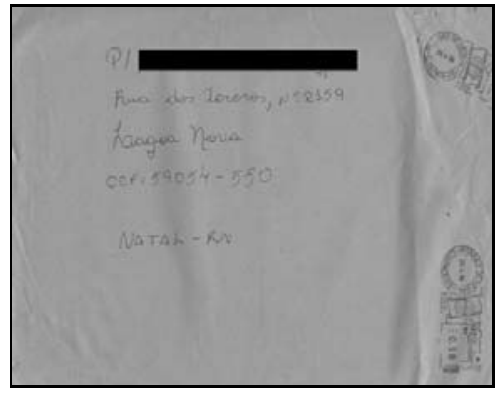

(a)

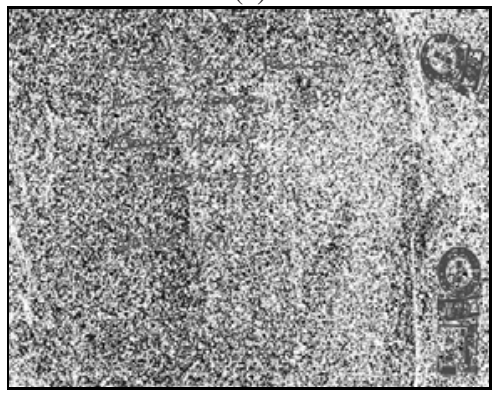

(c)

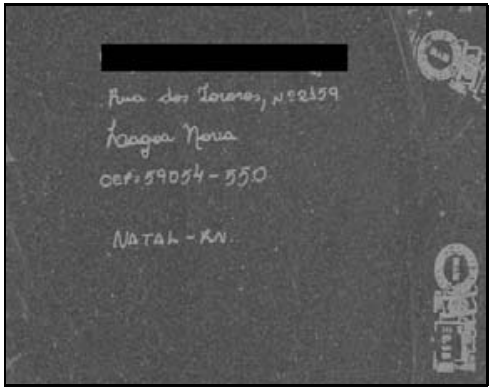

(b)

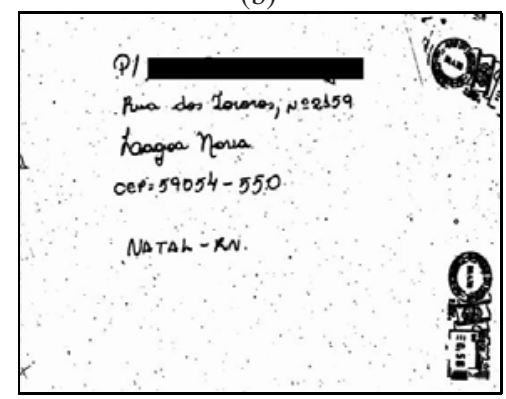

(d)

Fig. 3. Results of the proposed approach for an envelope image with creased background and no stamp: (a) Envelope image, (b) Fractal Image, (c) K-means clustering, (d) Handwritten address block and postmarks

clustered by K-means procedure. By comparing the obtained results with the ideal ones (ground-truth segmentation), it is possible to conclude that the proposed approach is robust if we consider the handwritten address block and postmark classes without a priori knowledge about the position of each semantic class. The $2 \mathrm{D}$ variation procedure was shown to be appropriate in our purpose. In spite of its simplicity, the centroid initialization in K-means was shown to be efficient in the segmentation challenge. In addition, since this method does not use a priori knowledge, it can be employed to segment other types of document images. Future works will focus segmentation of magazine envelopes with plastic covers. 


\section{Acknowledgments}

We would like to acknowledge support for this research from PUCPR, CNPq/MCT and the Brazilian Post Office Agency (Correios Brasileiros).

\section{References}

1. Yu, B., Jain, A., Mohiuddin, M.: Address block location on complex mail pieces. Technical Report MSUCPS:TR97-12, Dept. of Computer Science, Michigan State University (1997)

2. Yonekura, E., Facon, J.: Postal envelope segmentation by 2-d histogram clustering through watershed transform. ICDAR 2003, 7th International Conference on Document Analysis and Recognition 1 (2003) 338-342

3. Diayana, W.M., Larcher, J., Besar, R.: A comparison of clustered microcalcifications automated detection methods in digital mammogram. ICASSP (2003) 385-388

4. Ringler, A.: Texture segmentation by local fractal dimension as applied to oceanic search and rescue. IEEE ICICS, International Conference on Information Communications Signal Processing 2 (2003) 975-979

5. Samarbandu, J., Acharya, R., Hausmann, E., Allen, K.: Analysis of bone x-rays using morphological fractals. IEEE Transactions on Medical Imaging 12 (1993) 466-474

6. Chaudhuri, B., Sarkar, N., Kundu, P.: Improved fractal geometry based texture segmentation technique. IEE Proceedings 140 (1993) 233-241

7. Dubuc, B., Quiniuo, J., Roques-Carmes, C., Tricot, C., Zucker, S.: Evaluating the fractal dimensions of profile. Phys. Rev. 39 (1989) 1500-1512

8. Bishop, C.: Neural Networks for Pattern Recognition. Oxford University Press (1995)

9. Jain, A., Dubes, R.: Algorithms for Clustering Data. Prentice Hall: New Jersey (1988)

10. Fayad, U., Reina, C., Bradley, P.: Initialization of iterative refinement clustering algorithms. 4th KDD98- International Conference on Knowledge Discovery and Data Mining (1998) 